Check for updates

Cite this: RSC Adv., 2019, 9, 8926

\title{
A UPLC-MS-based metabolomics approach to reveal the attenuation mechanism of Caowu compatibility with Yunnan Baiyao $\uparrow$
}

\author{
Jun-ling Ren, ${ }^{a}$ Hui Sun, ${ }^{* a}$ Hui Dong, ${ }^{a}$ Le Yang, ${ }^{a}$ Ai-hua Zhang, ${ }^{a}$ Ying Han, ${ }^{a}$ Li Wang, ${ }^{a}$ \\ Liang Liub and Xi-jun Wang (DD*ab
}

Yunnan Baiyao (YNBY) is a well-known traditional Chinese medicine containing Caowu (Aconiti kusnezoffii radix, CW). However, the application of YNBY is limited by the toxicity of CW. Notably, CW is not used alone in YNBY, but is combined with other herbs in a formula for clinical use. In the present study, the compatibility of the protective effects and mechanism of YNBY with the potential toxicity of CW was investigated. After combining with other compatible herbs, the serum metabolic disorder induced by CW can be regulated. Using UPLC-MS-based metabolomics, 63 endogenous serum metabolites were identified as being associated with the potential toxicity of CW, 17 of which were regulated to normal levels when CW was combined with other compatible herbs in YNBY. These regulated metabolites were closely related to glycerophospholipid metabolism, glycosylphosphatidylinositol (GPI)-anchor biosynthesis, tyrosine metabolism, and primary bile acid biosynthesis metabolic pathways. This study aims to evaluate the attenuation mechanism of CW compatibility with YNBY.

Received 1st December 2018 Accepted 5th March 2019

DOI: $10.1039 / \mathrm{c} 8 \mathrm{ra09894h}$

rsc.li/rsc-advances investigated. Research into the toxicity of YNBY is of international interest and urgently needed.

As an emerging "omics" of the post-genome era, metabolomics has become an increasingly indispensable technology in life science research. ${ }^{9,10}$ This approach has seen broad applications, such as in therapeutic effect evaluation, ${ }^{11-13}$ biomarker discovery, ${ }^{14,15}$ medicine quality control, ${ }^{16-18}$ and toxicity assessment. ${ }^{19-21}$ The effect of YNBY as a formula on metabolism might be complex and multidimensional. Accordingly, the effect of YNBY system interference on metabolism might be assessed using a metabolomics-based strategy, in agreement with the holistic concept of TCM. ${ }^{22}$

Herein, a UPLC-MS-based metabolomics platform was established to determine the toxicity attenuation effect resulting from compatibility with YNBY. Serum was collected from healthy rats and metabolic data were used to evaluate the potential toxicity of $\mathrm{CW}$, processed CW (ZCW), YNBY, and YNBY lacking CW (QCW), as well as the possible detoxication mechanism. The information gained from the present study will provide a good foundation for further rational clinical applications of YNBY.

\section{Materials and methods}

${ }^{a}$ National Chinmedomics Research Center, Sino-America Chinmedomics Technology Collaboration Center, National TCM Key Laboratory of Serum Pharmacochemistry, Chinmedomics Research Center of TCM State Administration, Laboratory of Metabolomics, Department of Pharmaceutical Analysis, Heilongjiang University of Chinese Medicine, Heping Road 24, Harbin 150040, China. E-mail: xijunwangls@ 126.com; Fax: +86-451-82110818; Tel: +86-451-82110818

${ }^{b}$ State Key Laboratory of Quality Research in Chinese Medicine, Macau University of Science and Technology, Avenida Wai Long, Taipa, Macau, China

$\dagger$ Electronic supplementary information (ESI) available. See DOI: 10.1039/c8ra09894h

\section{Chemicals and reagents}

Acetonitrile and methanol (HPLC grade) were purchased from Fisher Scientific Corporation (Loughborough, UK). Deionized water was produced using a Milli-Q Ultra-pure water system (Millipore, Billerica, USA). Formic acid (HPLC grade, FA) was provided by Honeywell Company (USA). Drug powders of CW, ZCW, QCW, and YNBY (these powders contain excipients and 
the same CW contents) were provided by YNBY Group Corporation.

\begin{abstract}
Animals
Male SD rats $(n=60$, clean grade, weight $=180-200 \mathrm{~g}, 8$ weeks old) from Liaoning Changsheng Biotechnology Co., Ltd. (Benxi, Liaoning, China) were used in the study. The room temperature was regulated at $21 \pm 5{ }^{\circ} \mathrm{C}$ with $60 \pm 5 \%$ humidity. The rats were subjected to a $12 \mathrm{~h}$ light-dark cycle and had free access to a standard diet and water. The animals were allowed to acclimatize in metabolism cages for 1 week prior to dosing. After acclimatization, the animals were randomly divided into five treatment groups of 12 rats each, named as follow: control group, CW group, ZCW group, YNBY group, and QCW group. Rats in the drug groups were orally administrated at a dose of $180 \mathrm{mg} \mathrm{kg}{ }^{-1}$ and $1 \mathrm{~mL} / 100 \mathrm{~g}$ of rat weight for 15 consecutive days (Fig. 1). Rats in the control group were orally administrated with distilled water of equal dose for 15 consecutive days. The study was approved by the Ethical Committee of Heilongjiang University of Chinese Medicine and was conducted according to the principles expressed in the Declaration of Helsinki.
\end{abstract}

\section{Sample collection and preparation}

On day 16 , after the rats were orally administrated for $1 \mathrm{~h}$, blood was collected from the hepatic portal vein. After standing at room temperature for $1 \mathrm{~h}$, the blood was centrifuged at $3000 \mathrm{rpm}$ for $10 \mathrm{~min}$ at $4{ }^{\circ} \mathrm{C}$. The serum was then collected and frozen at $-80{ }^{\circ} \mathrm{C}$ until analysis. Before all rats were sacrificed, the heart, liver, spleen, lungs, and kidneys were quickly removed and fixed in $10 \%$ formalin for $24 \mathrm{~h}$, stained with hematoxylin and eosin (H\&E), and then observed by visible light microscopy and photographed.

Thawed serum $(100 \mu \mathrm{L})$ was mixed with methanol $(400 \mu \mathrm{L})$ and vortexed for $1 \mathrm{~min}$ to achieve protein precipitation,

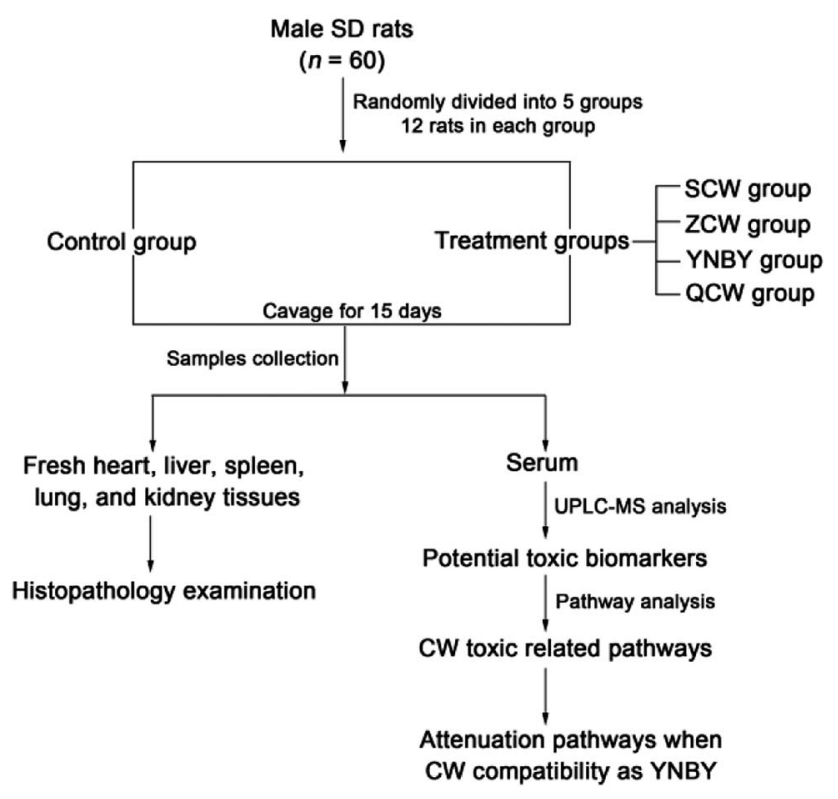

Fig. 1 Experimental design. followed by centrifugation at $13000 \mathrm{rpm}$ for $15 \mathrm{~min}$ at $4{ }^{\circ} \mathrm{C}$. Some of the supernatant $(350 \mu \mathrm{L})$ was dried under nitrogen and redissolved in $50 \%$ methanol $(100 \mu \mathrm{L})$. After vortexing for $1 \mathrm{~min}$ and centrifuging at $13000 \mathrm{rpm}$ for $15 \mathrm{~min}$ at $4{ }^{\circ} \mathrm{C}$, the supernatant was transferred to a glass vial for analysis.

\section{Chromatographic conditions}

Chromatographic separation was performed using a Waters ACQUITY UPLC system (Waters Corporation, Milford, MA). An 2 $\mu \mathrm{L}$ aliquot of the serum sample solution was injected into an ACQUITY UPLC HSS T3 column $(100 \mathrm{~mm} \times 2.1 \mathrm{~mm}, 1.8 \mu \mathrm{m}$; Waters Corporation, Milford, MA) at $35{ }^{\circ} \mathrm{C}$ with a flow rate of 0.4 $\mathrm{mL} \min ^{-1}$. The optimal mobile phase containing $0.1 \%$ formic acid in acetonitrile (solvent $\mathrm{A}$ ) and $0.1 \%$ formic acid in water (solvent B) was eluted using a linear gradient elution program, as follows: $0 \mathrm{~min}$ at $1 \% \mathrm{~A} ; 0-2.0 \mathrm{~min}$ at $1-30 \% \mathrm{~A} ; 2.0-3.0 \mathrm{~min}$ at $30-40 \% \mathrm{~A} ; 3.0-4.0 \mathrm{~min}$ at $40-50 \% \mathrm{~A} ; 4.0-5.0 \mathrm{~min}$ at $50-60 \% \mathrm{~A}$; $5.0-6.0 \mathrm{~min}$ at $60-65 \% \mathrm{~A} ; 6.0-7.0 \mathrm{~min}$ at $65-70 \% \mathrm{~A} ; 7.0-10.0 \mathrm{~min}$ at $70-99 \% \mathrm{~A} ; 10.0-12.0 \mathrm{~min}$ at $99 \% \mathrm{~A}$.

\section{Mass spectrometry conditions}

High-definition mass spectrometry was performed on Micromass Q-TOF micro Synapt High Definition Mass Spectrometer (Synapt HDMS, Waters, Manchester, UK) equipped with electrospray ionization (ESI) in both positive and negative ion modes. The optimal conditions of analysis were as follows: (i) for $\mathrm{ESI}^{+}$: source temperature, $110{ }^{\circ} \mathrm{C}$; capillary voltage, $3 \mathrm{kV}$; cone voltage, $30 \mathrm{~V}$; extraction cone voltage, $5.0 \mathrm{~V}$; desolvation temperature, $350{ }^{\circ} \mathrm{C}$; cone gas flow, $50 \mathrm{~L} \mathrm{~h}^{-1}$; desolvation gas flow, $800 \mathrm{~L} \mathrm{~h}^{-1}$; (ii) for $\mathrm{ESI}^{-}$: source temperature, $110{ }^{\circ} \mathrm{C}$; capillary voltage, $2.5 \mathrm{kV}$; cone voltage, $30 \mathrm{~V}$; extraction cone voltage, $5.0 \mathrm{~V}$; desolvation temperature, $350{ }^{\circ} \mathrm{C}$; cone gas flow, $50 \mathrm{~L} \mathrm{~h}^{-1}$; desolvation gas flow, $800 \mathrm{~L} \mathrm{~h}^{-1}$. Data were acquired using an external reference (LockSpray) consisting of a $1 \mathrm{ng} \mathrm{\textrm {L } ^ { - 1 }}$ solution of leucine enkephalin infused at a flow rate of 5 $\mathrm{L} \min ^{-1}$ via a LockSpray interface, which generated a reference ion for positive $\left([\mathrm{M}+\mathrm{H}]^{+}=556.2771\right)$ and negative $\left([\mathrm{M}-\mathrm{H}]^{-}=\right.$ $554.2615)$ ion modes to ensure accurate MS analysis.

\section{Data processing and multivariate data analysis}

The acquired UPLC-MS data were imported to Progenesis QI for peak picking, alignment, normalized, and were then introduced to EZinfo 2.0 software for principal component analysis (PCA) and partial least squares discriminant analysis (PLS-DA). Student's $t$-test was performed to identify the metabolic difference between groups and screen the metabolites with variable importance in projection (VIP) values $>1$ as the differential metabolites. The MetaboAnalyst platform (http:// www.metaboanalyst.ca/) was used to analyze the relative metabolic pathways associated with potential toxicity. In all cases, a $p$ value $<0.05$ was considered to be statistically significant.

\section{Identification of biomarkers}

Differential metabolites were compared with theoretical accurate molecular masses and matched with databases, including 
HMDB, METLIN, and Lipid Maps, with mass tolerances of less than $5 \mathrm{ppm}$. Potential toxic biomarkers were finally confirmed by MS/MS for the characteristic ions and fragmentation patterns.

\section{Results}

\section{Histopathology evaluation}

From histopathological observations (Fig. S1†), there was no significant change in the microscopic histology of H\&E stained in each organ. The histopathological results showed that no obvious toxicity was found in each administration group within a treatment cycle.

\section{Multivariate statistical analysis of metabolite profiling}

Raw data from UPLC-MS were analyzed by Progenesis of QI software and, after peak alignment and detection, the results were exported into EZinfo 2.0 software for multivariate statistical analysis. The data were first analyzed by PCA, which showed no clustering trend between each group. Owing to the prospective metabolomics, PLS-DA was performed to make differences among the groups more obvious, with a clear classification between the clustering of each group identified (Fig. 2). The PLS-DA score plot showed that compared with other groups, CW group was far from the control group, followed by the ZCW and QCW groups, while the YNBY group was closest to the control group. This suggested that CW may have potential toxic effects on normal rats during an administration cycle, and that the potential toxic effect was significantly reduced by compatibility with YNBY.

\section{Metabolite identification and metabolic pathway analysis}

The VIP of PLS-DA was performed to identify differential ions with larger contributions between the control group and CW group, which were usually regarded as potential biomarkers. Metabolite identification was conducted using high-resolution MS and MS/MS fragments, and website database analyses. Based on the above protocol, a total of 63 ions (in both positive and negative ion modes) were identified, which contributed to classification of the control group and CW group (Table $\mathrm{S} 1 \dagger$ ). Furthermore, a heatmap was constructed according to the relative abundance of the above differential metabolites to visualize differences among different treated groups (Fig. 3). Cluster analysis showed that the levels of metabolites in the control
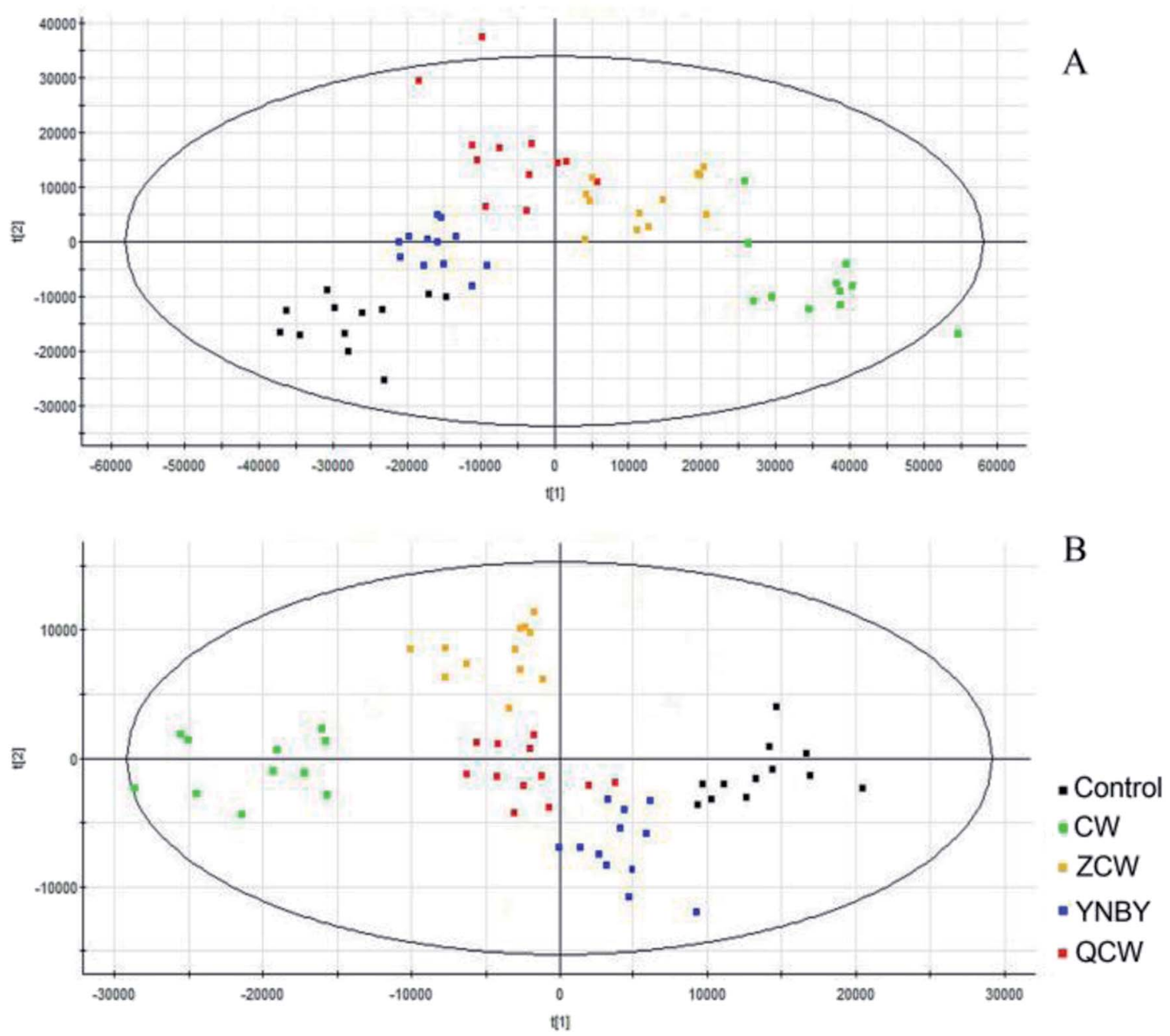

Fig. 2 PLS-DA of control, CW, ZCW, YNBY, and QCW groups based on the serum metabolic profiles in both positive and negative ion modes. 


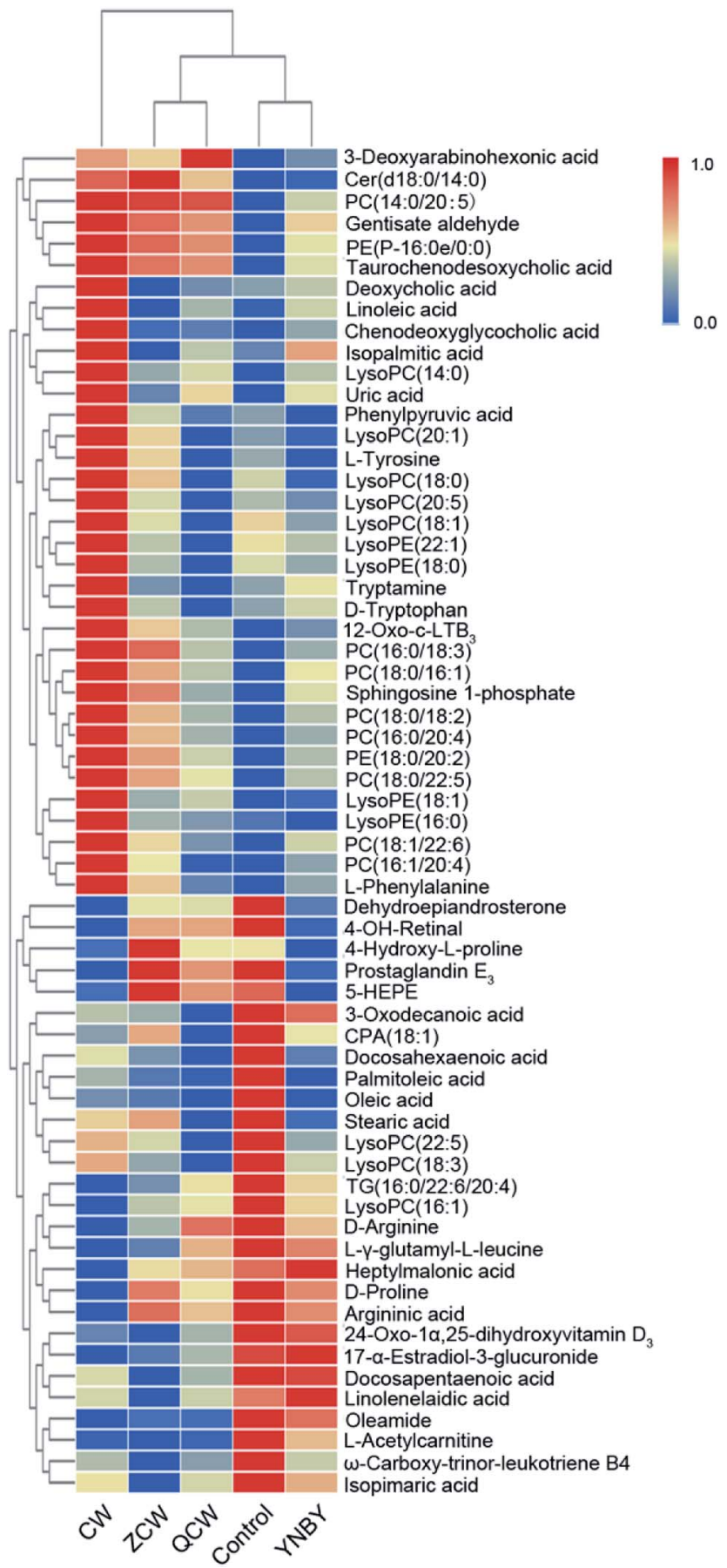

Fig. 3 Heatmap visualizing changes in the relative content values of potential toxic biomarkers in the control, CW, ZCW, YNBY, and QCW groups.

group were similar to those in the YNBY group, with almost no similar trends between the CW group and other groups.

To further elucidate the possible metabolic pathway affected by $\mathrm{CW}$, the above potential differential metabolites were introduced into MetaboAnalyst software. The related pathway with a higher score is shown in Fig. 4, which contained linoleic acid metabolism, D-arginine and D-ornithine metabolism, glycerophospholipid metabolism, phenylalanine metabolism, arginine and proline metabolism, tyrosine metabolism,

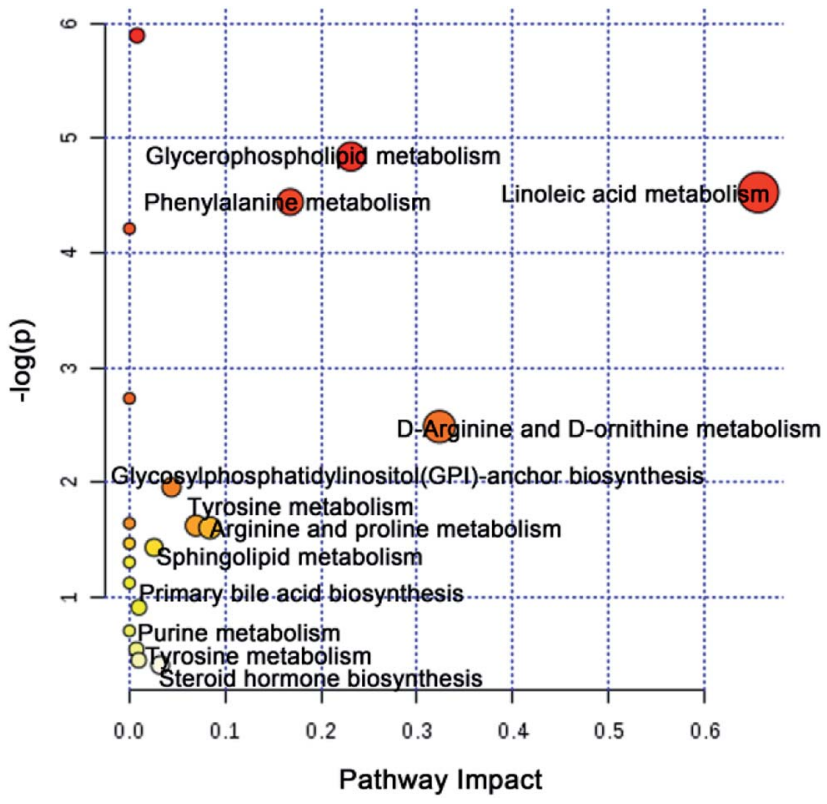

Fig. 4 Metabolic pathways analysis based on potential toxic biomarkers of $\mathrm{CW}$.

glycosylphosphatidylinositol (GPI)-anchor biosynthesis, steroid hormone biosynthesis, sphingolipid metabolism, primary bile acid biosynthesis, purine metabolism, and tyrosine metabolism.

\section{Locking of core attenuated biomarkers and pathways}

To further explore the attenuation effect of $\mathrm{CW}$ due to compatibility with YNBY, the relative contents of the potential toxic biomarkers in each group were compared to explicitly determine the core potential toxic biomarkers. Using the contents of potential toxic biomarkers in the control group as reference, the relative contents of potential toxic biomarkers in the $\mathrm{CW}$ group showed the largest deviation, while other groups showed contents close to those of the control group. Finally, the attenuation effect of YNBY on CW was found to be mediated by the following 17 metabolites: TG(16:0/22:6/20:4), PC(14:0/20:5), PC(16:0/18:3), PC(16:0/20:4), PC(18:0/22:5), PE(18:0/20:2), PE(P16:0e/0:0), lysoPE(16:0), lysoPE(18:1), lysoPC(18:0), L-acetylcarnitine, 12-oxo-c-LTB 3 , heptylmalonic acid, L- $\gamma$-glutamyl-Lleucine, gentisate aldehyde, taurochenodeoxycholic acid, and 17- $\alpha$-estradiol-3-glucuronide (Fig. 5). These biomarkers are closely related to four metabolic pathways, namely, glycerophospholipid metabolism, GPI-anchor biosynthesis, tyrosine metabolism, and primary bile acid biosynthesis (Fig. 6), which were the core metabolic pathways of $\mathrm{CW}$ attenuation due to compatibility with YNBY.

\section{Discussion}

YNBY is extensively used in traumatology, including to decrease bleeding and clotting times, as an anti-inflammatory, and to heal wounds, for more than a century ${ }^{23,24}$ However, owing to the well-known toxicity of CW, which is found in YNBY, the clinical application of YNBY has been controversial. In this study, no 


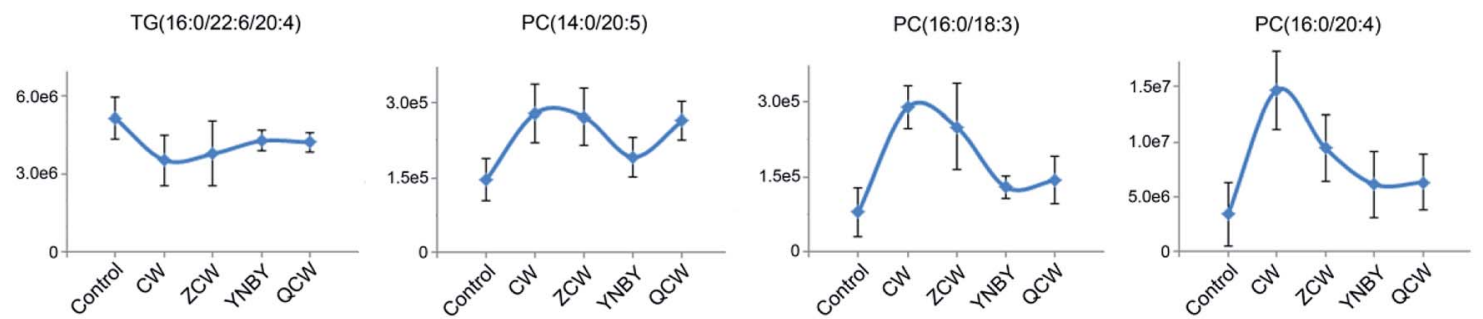

PC(18:0/22:5)
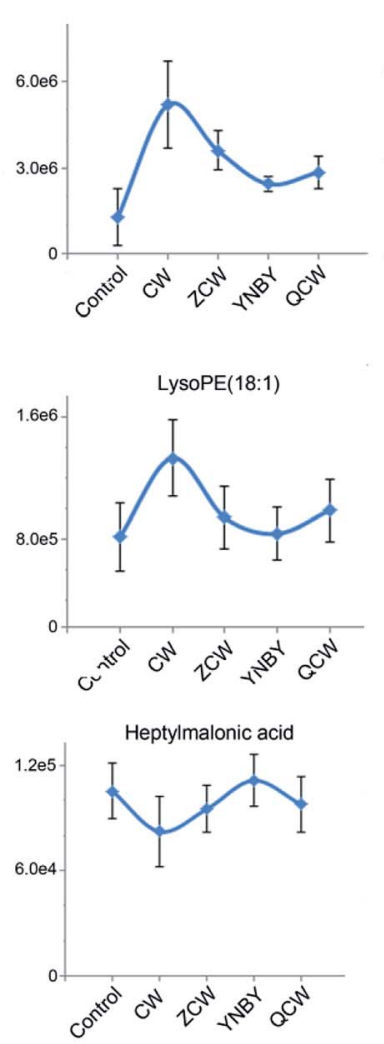

17-alpha-Estradiol-3 glucuronide

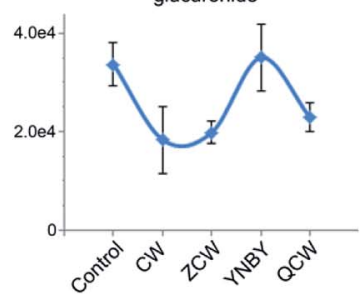

PE(18:0/20:2)
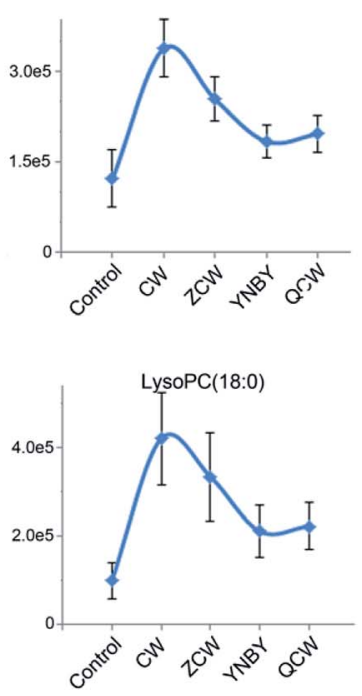

L-y-glutamyl-L-leucine

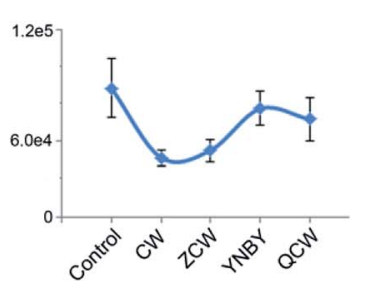

PE(P-16:0e/0:0)
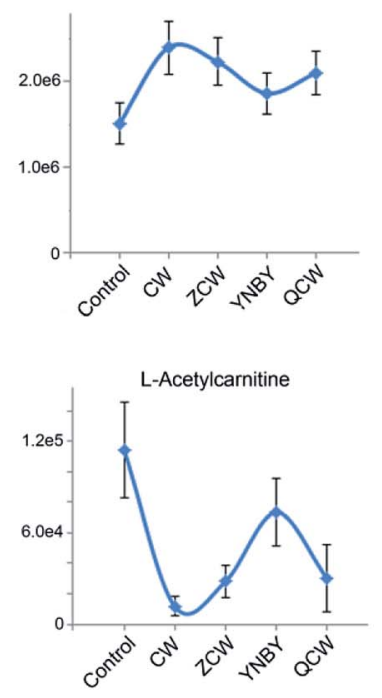

Gentisate aldehyde

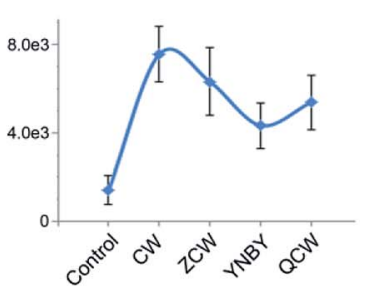

LysoPE(16:0)
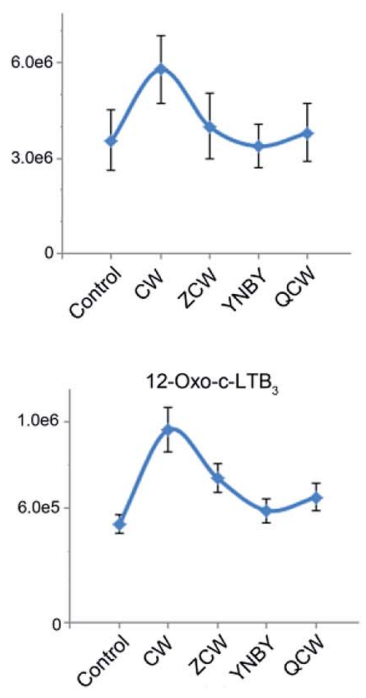

Taurochenodesoxycholic acid

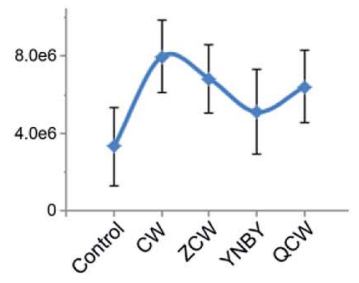

Fig. 5 Changes in core potential toxic biomarkers in the control, CW, ZCW, YNBY, and QCW groups.

toxicity was found in either the CW group or YNBY group within a clinical medication cycle.

In recent years, an increasing number of toxicity studies have adopted an metabolomics approach. Metabolomics is an unbiased analysis of metabolites to detect subtle dynamic changes and potential adverse effects. ${ }^{25-32}$ Using metabolomics, we determined the potential toxicity in CW group. The PLS-DA results showed that there were significant differences in the serum metabolites between the control group and CW or ZCW groups, which might be due to toxicity (Fig. 2). Meanwhile, the metabolic profile of the YNBY group was most similar to that of the control group, followed by the QCW group. This result showed that, owing to compatibility with other herbs in YNBY, the toxicity of CW was reduced or even disappeared. In the course of preparing the prescription, the quality and quantity of CW and the toxicokinetics might change due to interactions with herbs and the influence of preparation processes. Therefore, the toxicity of $\mathrm{CW}$ might decrease or disappear, which might be mainly due to the attenuation effect of prescription compatibility. 


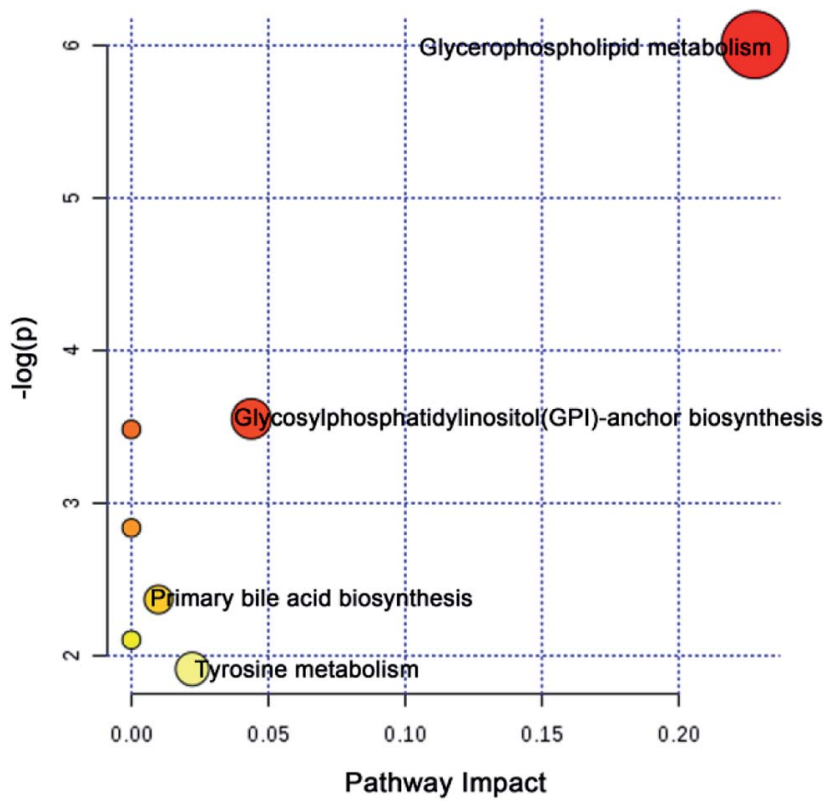

Fig. 6 Core attenuated metabolic pathways based on the core potential toxic biomarkers of CW.

The potential toxicity mainly caused disorders in 63 metabolites, including linoleic acid metabolism, and 11 metabolic pathways (Fig. 4). According to the performance of differential metabolites in different treatment groups, core regulated potential toxic biomarkers and attenuated metabolic pathways were locked. As shown in Fig. 6, glycerophospholipid metabolism, GPI-anchor biosynthesis, tyrosine metabolism, and primary bile acid biosynthesis were the key metabolic pathways that played detoxification roles for YNBY compatibility.

Phosphatidylcholine (PC) is among the most abundant phospholipids in biological membranes, and forms the cell membrane bilayer structure with other phospholipids, which regulates membrane integrity and fluidity. ${ }^{33}$ PC has hepatoprotective effects and can resist liver injury caused by alcohol, drugs, and environmental pollutants. ${ }^{34,35}$ The significant increase in PC content might indicate that PC exerts a protective effect on liver cells against the toxic effects of CW. In a chronic pathophysiological stressed heart, a decreased triacylglycerol (TG) content can buffer potential toxic lipid intermediates in cells, and TG can also directly participate in the regulation of intracellular signaling pathways. ${ }^{36}$

Taurochenodeoxycholic acid is a main bioactive substance in animal bile acids that shows good anti-inflammatory activity. ${ }^{37}$ Taurochenodeoxycholic acid might significantly inhibit acute myocardial infarction and chronic inflammation by activating protease cascades to induce apoptosis. ${ }^{38,39}$ In this study, taurochenodeoxycholic acid was significantly increased in the CW group. After processing and compatibility, the content tended to be normal, suggesting that the potential inflammation was inhibited by processing and compatibility. Therefore, the attenuation effect of CW based on YNBY compatibility probably inhibited inflammation. Furthermore, as the downstream product of arachidonic acid, 12-oxo-c- $\mathrm{LTB}_{3}$ also participates in the inflammatory response. ${ }^{40}$ The increased level of 12 -oxo-c-
$\mathrm{LTB}_{3}$ also indicated that inflammation might be the key biological effect of detoxification in YNBY.

Gentisate aldehyde is the substrate in aldehyde oxidase 1, leucine and isoleucine degradation, tyrosine metabolism, tryptophan metabolism, vitamin $\mathrm{B}_{6}$ metabolism, nicotinic acid, and nicotinamide metabolism. Disorders of amino acid metabolism exist in various diseases, such as fungus, ${ }^{41}$ cancers,,${ }^{42,43}$ and diabetes, ${ }^{44}$ and have been regarded as potential targets for disease treatment. Amino acid metabolism disturbance is mainly manifested in the function of digestion and absorption of host cells, the biosynthesis of non-essential amino acids in the liver, and the oxidative decomposition of amino acids in tissues. The increased level of gentisate aldehyde suggested that CW had a potential effect on the liver.

17 - $\alpha$-Estradiol-3-glucosidate is the metabolite of 17 - $\alpha$-estradiol produced by UDP glucosyltransferase in the liver. Glutaraldehyde acidification helps discharge toxic substances, drugs, or other substances that cannot be used as body energy sources. ${ }^{45}$ Glucuronic acid is linked to other substances by glycosidic bonds, and the resulting glucuronides are much more water-soluble than the original substances, facilitating kidney excretion.$^{46}$ Compared with the control group, the 17- $\alpha$-estradiol-3-glucuronide content in the $\mathrm{CW}$ group was significantly lower, and gradually recovered after processing and compatibility with YNBY, indicating that glutaraldehyde acidification gradually became normal during these processes, which might help excrete toxic substances.

The present study also has limitations. Healthy rats were used as research objects, but future studies should be conducted using corresponding animal models to obtain better explanations. Other techniques, such as gas chromatography mass spectrometry, should be employed together with UPLC-MS to characterize more metabolites and clearly elucidate the mechanism.

\section{Conclusions}

This study showed, for the first time, that CW toxicity is attenuated by compatibility with YNBY using a UPLC-MS-based metabolomics platform. A total of 63 potential toxicity biomarkers and 12 related pathways were identified. Consequently, 17 core toxicity biomarkers and 4 core attenuated metabolic pathways were identified. PLS-DA analysis and core toxicity biomarkers both indicated that the YNBY prescription had a minimal effect on metabolites. As a possible mechanism, YNBY might exert its attenuation effects through inflammatory inhibition. The study provides information to improve the safety and rationality of clinical medication using YNBY.

\section{Conflicts of interest}

The authors declare no competing financial interests.

\section{Abbreviations}

$\begin{array}{ll}\text { CW } & \text { Caowu } \\ \text { ESI } & \text { Electrospray ionization } \\ \text { GPI } & \text { Glycosylphosphatidylinositol }\end{array}$


H\&E Hematoxylin and eosin

PLS-DA Partial least squares discriminant analysis

PC Phosphatidylcholine

PCA Principal components analysis

QCW Yunnan Baiyao without Caowu

TCM Traditional Chinese medicine

TG Triacylglycerol

VIP Variable importance in projection

YNBY Yunnan Baiyao

ZCW Processed Caowu

\section{Acknowledgements}

This work was supported by grants from the Key Program of Natural Science Foundation of State (Grant No. 81830110, 81430093, 81373930, 81673586, 81703685, 81302905, 81503386), National Key Subject of Drug Innovation (Grant No. 2015ZX09101043-005, 2015ZX09101043-011), TCM State Administration Subject of Public Welfare (Grant No. 2015468004), Major Projects of Application Technology Research and Development Plan in Heilongjiang Province (GX16C003), Young Talent Lift Engineering Project of China Association of Traditional Chinese Medicine (QNRC2-B06), Outstanding Talents Foundation of Heilongjiang University of Chinese Medicine (2018jc01), Doctoral Innovation Fund of Heilongjiang University of Chinese Medicine (2018bs02), University Nursing Program for Young Scholars with Creative Talents in Heilongjiang Province (UNPYSCT-2015118, UNPYSCT-2016213, UNPYSCT-2016212), Application Technology and Development of Youth Talents Project in Harbin (2014RFQXJ116), Chinese Postdoctoral Science Foundation (2017M621319b), Returned Oversea Scholars Program of Heilongjiang Province (2017QD0025), and Natural Science Foundation of Heilongjiang Province of China (QC2018117).

\section{References}

$1 \mathrm{~W}$. Cordier and V. Steenkamp, Herbal remedies affecting coagulation: a review, Pharm. Biol., 2012, 50(4), 443-452.

2 S. L. Ness, A. H. Frye, T. J. Divers, et al., Randomized placebocontrolled study of the effects of Yunnan Baiyao on hemostasis in horses, Am. J. Vet. Res., 2017, 78(8), 969-976.

3 C. W. Ogle, S. Dai and J. C. Ma, The haemostatic effects of the Chinese herbal drug Yunnan Bai Yao: a pilot study, Am. J. Chin. Med., 1976, 4(2), 147-152.

4 G. I. Solyanik, A. G. Fedorchuk, O. N. Pyaskovskaya, et al., Anticancer activity of aconitine-containing herbal extract BC1, Exp. Oncol., 2004, 26(4), 307-311.

5 H. Khan, S. M. Nabavi, A. Sureda, et al., Therapeutic potential of songorine, a diterpenoid alkaloid of the genus Aconitum, Eur. J. Med. Chem., 2018, 153, 29-33.

6 M. F. Chen, C. H. Ho, T. N. Tsai, et al., Amiodarone for the Successful Management of Caowu Poisoning - Induced Cardiac Arrhythmia, Acta Cardiol Sin., 2018, 34(2), 189-191.

7 Y. Yan, A. H. Zhang, H. Dong, et al., Toxicity and Detoxification Effects of Herbal Caowu via Ultra
Performance Liquid Chromatography/Mass Spectrometry Metabolomics Analyzed using Pattern Recognition Method, Pharmacogn. Mag., 2017, 13(52), 683-692.

8 G. Cao, Q. Li, H. Cai, et al., Investigation of the Chemical Changes from Crude and Processed Paeoniae Radix AlbaAtractylodis Macrocephalae Rhizoma Herbal Pair Extracts by Using Q Exactive High-Performance Benchtop Quadrupole-Orbitrap LC-MS/MS, J. Evidence-Based Complementary Altern. Med., 2014, 170959.

9 C. Jang, L. Chen and J. D. Rabinowitz, Metabolomics and Isotope Tracing, Cell, 2018, 173(4), 822-837.

10 A. H. Zhang, H. Sun and X. J. Wang, Mass spectrometrydriven drug discovery for development of herbal medicine, Mass Spectrom. Rev., 2018, 37(3), 307-320.

11 A. H. Zhang, Q. Liu, H. Zhao, et al., Phenotypic characterization of nanshi oral liquid alters metabolic signatures during disease prevention, Sci. Rep., 2016, 6, 19333.

12 H. Sun, A. H. Zhang, S. Qi, et al., Functional metabolomics discover pentose and glucuronate interconversion pathway as promising targets of Yanghuang syndrome treatment with Yinchenhao Tang, RSC Adv., 2018, 8, 36831-36839.

13 H. Sun, A. H. Zhang, S. B. Liu, et al., Cell metabolomics identify regulatory pathways and targets of magnoline against prostate cancer, J. Chromatogr. B: Anal. Technol. Biomed. Life Sci., 2018, 1102-1103, 143-151.

14 L. Cui, H. Lu and Y. H. Lee, Challenges and emergent solutions for LC-MS/MS based untargeted metabolomics in diseases, Mass Spectrom. Rev., 2018, 37(6), 772-792.

15 C. Liu, W. J. Zong, A. H. Zhang, et al., Lipidomic characterisation discovery for coronary heart disease diagnosis based on high-throughput ultra-performance liquid chromatography and mass spectrometry, RSC Adv., 2018, 8, 647-654.

16 A. D. Kennedy, B. M. Wittmann, A. M. Evans, et al., Metabolomics in the Clinic: A Review of the Shared and Unique Features of Untargeted Metabolomics for Clinical Research and Clinical Testing, J. Mass Spectrom., 2018, 53(11), 1143-1154.

17 X. J. Wang, A. H. Zhang, L. Kong, et al., Rapid discovery of quality-markers from Kaixin San using chinmedomics analysis approach, Phytomedicine, 2017, 54, 371-381.

18 H. Sun, A. H. Zhang, L. Yang, et al., High-throughput chinmedomics strategy for discovering the quality-markers and potential targets for Yinchenhao decoction, Phytomedicine, 2019, 54, 328-338.

19 M. Cuykx, R. M. Rodrigues, K. Laukens, et al., In vitro assessment of hepatotoxicity by metabolomics: a review, Arch. Toxicol., 2018, 92(10), 3007-3029.

20 H. Dong, A. H. Zhang, H. Sun, et al., Ingenuity pathways analysis of urine metabolomics phenotypes toxicity of Chuanwu in Wistar rats by UPLC-Q-TOF-HDMS coupled with pattern recognition methods, Mol. BioSyst., 2012, 8(4), 1206-1221.

21 L. Wang, H. Dong, A. H. Zhang, et al., Exploring the detoxification effects and mechanism of Caowu in 
prescription using liquid chromatography-high-resolution mass spectrometry-based metabolomics, Open Journal of Proteomics and Genomics, 2018, 3(1), 11-23.

22 A. H. Zhang, H. Sun, Z. Wang, et al., Metabolomics: towards understanding traditional Chinese medicine, Planta Med., 2010, 76(17), 2026-2035.

23 J. Frederick, S. Boysen, C. Wagg, et al., The effects of oral administration of Yunnan Baiyao on blood coagulation in beagle dogs as measured by kaolin-activated thromboelastography and buccal mucosal bleeding times, Can. J. Vet. Res., 2017, 81, 41-45.

24 H. He, X. Ren, X. Wang, et al., Therapeutic effect of Yunnan Baiyao on rheumatoid arthritis was partially due to regulating arachidonic acid metabolism in osteoblasts, $J$. Pharm. Biomed. Anal., 2012, 59, 130-137.

25 A. Zhang, H. Sun and X. Wang, Urinary metabolic profiling of rat models revealed protective function of scoparone against alcohol induced hepatotoxicity, Sci. Rep., 2014, 4, 6768.

26 H. Sun, H. Wang, A. Zhang, et al., Berberine ameliorates nonbacterial prostatitis via multi-target metabolic network regulation, OMICS: J. Integr. Biol., 2015, 19(3), 186-195.

27 A.-H. Zhang, H. Sun, G.-Li Yan, et al., Chinmedomics: A Powerful Approach Integrating Metabolomics with Serum Pharmacochemistry to Evaluate the Efficacy of Traditional Chinese Medicine, Engineering, 2019, 5, 60-68.

28 H. Sun, A. Zhang, Q. Song, et al., Functional metabolomics discover pentose and glucuronate interconversion pathways as promising targets for Yang Huang syndrome treatment with Yinchenhao Tang, RSC Adv., 2018, 8(64), 36831-36839.

29 A. Zhang, S. Qiu, H. Sun, et al., Scoparone affects lipid metabolism in primary hepatocytes using lipidomics, Sci. Rep., 2016, 6, 28031.

30 Xi-J. Wang, J.-L. Ren, Ai-H. Zhang, et al., Novel Applications of Mass Spectrometry-based Metabolomics in Herbal Medicines and Its Active Ingredients: Current Evidence, Mass Spectrom. Rev., 2019, 9999, 1-23.

31 S. Qiu, A. Zhang, T. Zhang, et al., Dissect new mechanistic insights for geniposide efficacy on the hepatoprotection using multiomics approach, Oncotarget, 2017, 8(65), 108760-108770.

32 A. Zhang, H. Wang, H. Sun, et al., Metabolomics strategy reveals therapeutical assessment of limonin on nonbacterial prostatitis, Food Funct., 2015, 6(11), 3540-3549.

33 J. N. van der Veen, J. P. Kennelly, S. Wan, et al., The critical role of phosphatidylcholine and phosphatidylethanolamine metabolism in health and disease, Biochim. Biophys. Acta, Biomembr., 2017, 1859(9), 1558-1572.

34 Y. C. Kim, S. Seok, S. Byun, et al., AhR and SHP regulate phosphatidylcholine and S-adenosylmethionine levels in the one-carbon cycle, Nat. Commun., 2018, 9, 540.

35 T. A. Pagonis, G. N. Koukoulis, C. S. Hadjichristodoulou, et al., Multivitamins and phospholipids complex protects the hepatic cells from androgenic-anabolic-steroidsinduced toxicity, Clin. Toxicol., 2008, 46(1), 57-66.

36 A. N. Carley and E. D. Lewandowski, Triacylglycerol turnover in the failing heart, Biochim. Biophys. Acta, 2016, 1861(10), 1492-1499.

37 M. Wei, M. Q. Liu, G. Hong, et al., Taurochenodeoxycholic Acid Suppresses NF- $\kappa$ B Activation and Related Cytokines Expression in Peritoneal Macrophages from Adjuvant Arthritis Rat, Rec. Nat. Prod., 2018, 12(3), 263-272.

38 M. Liu, W. Mao, H. Guan, et al., Effects of taurochenodeoxycholic acid on adjuvant arthritis in rats, Int. Immunopharmacol., 2011, 11(12), 2150-2158.

39 X. Wang, Z. Zhang, X. He, et al., Taurochenodeoxycholic acid induces NR8383 cells apoptosis via PKC/JNK-dependent pathway, Eur. J. Pharmacol., 2016, 786(5), 109-115.

40 T. Okuno, M. A. Gijon, S. Zarini, et al., Altered eicosanoid production and phospholipid remodeling during cell culture, J. Lipid Res., 2018, 59, 542-549.

41 M. W. McCarthy and T. J. Walsh, Amino Acid Metabolism and Transport Mechanisms as Potential Antifungal Targets, Int. J. Mol. Sci., 2018, 19(3), 909.

42 Y. J. Cha, E. S. Kim and J. S. Koo, Amino Acid Transporters and Glutamine Metabolism in Breast Cancer, Int. J. Mol. Sci., 2018, 19(3), 907.

43 M. J. Lukey, W. P. Katt and R. A. Cerione, Targeting amino acid metabolism for cancer therapy, Drug Discovery Today, 2017, 22(5), 796-804.

44 Y. Hayashi and Y. Seino, Regulation of amino acid metabolism and alpha-cell proliferation by glucagon, $J$. Diabetes Invest., 2018, 9(3), 464-472.

45 R. Fujiwara, E. Yoda and R. H. Tukey, Species differences in drug glucuronidation: Humanized UDPglucuronosyltransferase 1 mice and their application for predicting drug glucuronidation and drug-induced toxicity in humans, Drug Metab. Pharmacokinet., 2018, 33(1), 9-16.

46 G. Yang, S. Ge, R. Singh, et al., Glucuronidation: driving factors and their impact on glucuronide disposition, Drug Metab. Rev., 2017, 49(2), 105-138. 